\title{
La medicina social y las experiencias de atención primaria de salud (APS) en Latinoamérica: historia con igual raíz*
}

\author{
Giovanni Apráez Ippolito
}

Universidad Nacional de Colombia, Bogotá, Colombia. Email: ngapraezi@unal.edu.co

Resumen: Los orígenes de la APS y de la Política de SALUD PARA TODOS se remontan al siglo XVIII y al movimiento de Medicina Social de 1848. Del siglo XX se destacan las experiencias pioneras de los años 30-50s. Entre ellas, Chile, Saskachewa (Canadá), EUA, Suráfrica, con Henry Sigerist, Sidney Kark, Salvador Allende, Gustavo Molina, trabajos pioneros de la medicina y la epidemiologia social vinculados a los del antropólogo Benjamin Paul. Así como personajes menos reconocidos en APS especialmente el italiano Franco Basaglia transformador de la atención psiquiátrica y sanitaria con los principios de libertad terapéutica. A éstas están articuladas numerosas y desconocidas experiencias en América Latina y Colombia con Héctor Abad Gómez, Santiago Rengifo y César Uribe Piedrahita por el proceso de "Renovación de APS" de la OPS. En Latinoamérica, las luchas por la salud estaban articuladas a la luchas por una sociedad justa, con personajes como Ernesto Guevara desde la medicina, Paulo Freire en pedagogía, teología y filosofía de la liberación, y el sociólogo colombiano Camilo Torres, que aportaron metodologías cualitativas en la investigación en salud, los que junto aportes de integración como el Desarrollo a Escala Humana, consideran a los servicios sanitarios como elementos principales en la vida de las personas, y aportan a la construcción transdisciplinar e intersectorial de la salud para nuestro hoy.

Palabras clave: APS, Salud para todos, Políticas Publicas, política de salud.

\section{Social medicine and the experiences on primary health care (PHC) in Latin America: history with the same root}

\footnotetext{
Abstract: The origins of the APS and the Policy HEALTH FOR ALL go back to the eighteenth century and the movement of Social Medicine on 1848. Twentieth century highlights the pioneering experiences of the years 30-50s. Among them, Chile, Saskatchewan (Canada), USA, South Africa, with Henry Sigerist, Sidney Kark, Salvador Allende, Gustavo Molina, pioneering work of social medicine and epidemiology related to the anthropologist Benjamin Paul. As well as less recognized characters, especially the Italian Franco Basaglia, APS who transformed psychiatric care and health with the principles of therapeutic freedom. To these are articulated numerous and unknown experiences in Latin America and Colombia with Héctor Abad Gómez, Santiago Rengifo and César Uribe Piedrahita by the process of PHC renewal» of the OPS. In Latin America, the struggles for health were articulated to the struggles for a just society, with people like Ernesto Guevara in medicine, Paulo Freire in pedagogy, theology and philosophy of liberation, and
} 
the sociologist Camilo Torres, who provided qualitative methodologies on health research, which altogether with views on integration such as Human Scale Development, consider health services as key elements in people's lives, and contribute in building cross-disciplinary and intersectorial health for our today.

Key words: APS, Health for all, Public Policy, health policy.

\title{
Medicina social e as experiências de atenção primária à saúde (APS) na América Latina: história com a mesma raiz
}

\begin{abstract}
Resumo: As origens da APS e da Política de Saúde PARA TODOS voltar ao século XVIII e do movimento de Medicina Social, 1848. século XX destaca as experiências pioneiras dos anos 30-50s. Incluindo o Chile, Saskatchewan (Canadá), EUA, África do Sul, com Henry Sigerist, Kark Sidney, Salvador Allende, Gustavo Molina, um trabalho pioneiro da medicina social e epidemiologia relacionada com o antropólogo Paul Benjamin. E menos reconhecidas personagens, especialmente o italiano Franco Basaglia transformador APS cuidados psiquiátricos e de saúde com os princípios da terapia de liberdade. Estes são articuladas inúmeras experiências e desconhecidos na América Latina e Colômbia, com Héctor Abad Gómez, Rengifo Santiago e César Piedrahita Uribe pelo processo de renovação da APS “da OPS. Na América Latina, a luta pela saúde foram articuladas com a luta por uma sociedade justa, com pessoas como Ernesto Guevara de medicina, Paulo Freire, em Pedagogia e Teologia e filosofia da libertação, incluindo o sociólogo Camilo Torres, que forneceram as metodologias qualitativas investigação em saúde com a participação de integração, Escala de Desenvolvimento Humano de considerar elementos-chave dos serviços de saúde na vida das pessoas e contribuindo na construção de inter-disciplinar e de saúde para o nosso hoje.
\end{abstract}

Palavras-chave: APS, Saúde para Todos, Políticas Públicas, Política de Saúde.

$$
* * *
$$

\section{APS y Pioneros de la Medicina Social}

La historia y el desarrollo de la Atención Primaria de Salud y de la Política de salud para todos en el año 2000 no parten de Alma Ata (Litsios 2002:17) como se lee en la mayoría de textos sino que tienen raíces en la "medicina social” con el movimiento liderado por Rudolf Virchow y Jules Guerin entre otros (Waitzkin 2006:31) en 1848, al que le anteceden experiencias del siglo XVIII, con Villermé, Johann Peter Frank (Sigerist 2006:28) y otros, que son reflejo del pensamiento en la construcción de la salud como derecho.

En el siglo XX la medicina social resurge con personas y experiencias concretas para la APS; Henry Sigerist lo hacia en 1939 luchando por la organización del Sistema de Salud en EUA y su influencia en Suráfrica donde recibió un doctorado honoris causa en Literatura en ese año. Sus escritos, permiten conocer valiosos documentos casi perdidos de J.P. Frank (Frank 1941:14) y un artículo de 1944 (Sigerist 2003:29), es antecedente fundamental en la formulación de la Política de Salud Todos en el año 2000 propuesta por la OMS en 1977; Sigerist participa en la experiencia de la 
Provincia de Saskachewa base del actual sistema de salud canadiense, también están sus influencias en las experiencias las de Suráfrica, EUA e Israel con los exiliados surafricanos liderados por Sidney Kark y que contó con personajes destacados como John Cassel, Mervin Susser, donde desarrollaron la APS de base comunitaria (COPC) y son trabajos pioneros de epidemiología social (Brown 2002:5) que a su influenciaron la importante experiencia con los Navajos realizada por McDermot y Dubois en EUA que buscaba dar atención en salud con un enfoque integral. Diversas experiencias como está y otras fueron recogidas en 1956 en el libro pionero de la antropología médica de Benjamin David Paul (Paul 1956:23).

El surgimiento de los Servicios Nacionales de Salud (SNS) en la postguerra como parte del Estado Bienestar, es tema fundamental en la Historia de la Medicina Social y en la garantía del acceso real a los servicios de salud, en donde se destacan el SNS Británico (Navarro 1984:22), al que Sigerist (Ibid 1943:30) visionara como cambio paradigmático en la organización de la Seguridad Social 5 años antes de su aparición; y el Chileno, el primero en toda América (1952), con antecedentes legislativos en los años de 1930, mucho antes de la postguerra, experiencia no se lo menciona ni tampoco a sus constructores donde es protagónico Salvador Allende (Molina 2006:20).

Uno de los personajes menos reconocidos en APS es el italiano Franco Basaglia pese a ser transformador de la atención psiquiátrica con los principios de libertad terapéutica e integración social influyendo determinantemente en la creación del Sistema Nacional de Salud Italiano en 1978. Sus trabajos tienen lugar en los años 60 desconocida por los documentos de APS, en el Hospital Siquiátrico de Gorizia en 1963 con más de 500 personas porque significó un cambio en la atención de la personas con enfermedad mental (Basaglia 1978:4), cuyo tratamiento, como es conocido, representa exclusión y violencia institucionalizada. Basaglia, al comprenderlo, consideró a los enfermos, personas reintegrando su poder para tomar decisiones sobre sí mismos: "los sacó de sus celdas, cortó sus camisas de fuerza e hizo la asamblea con ellos para decidir" (como lo afirma Giorgio Antonucci).

El cierre de los hospitales siquiátricos en Italia representa un símbolo del ejercicio ciudadano por la libertad, de superación de la exclusión social e hizo parte del movimiento que fundó el Sistema Nacional de Salud en Italia en 1978. La conmemoración de los 30 años de la ley Basaglia en 2008 permitieron la publicación en español de sus conferencias en Brasil dictadas en 1979.

\section{APS, Transdisciplinaridad y Cuestionamientos al Modelo Médico y de Desarrollo}

La década de los 70 enriquece la historia con los cuestionamientos de Thomas McKeown sobre los avances médicos y su impacto en mejorar las condiciones de vida, se suman Michael Foucault con sus 
cuestionamientos sobre la medicalización e Illich con el polémico libro “Nemesis Médica”, incluye autores clásicos hoy, como Dubois, Chocrane, Morin y Cassel, ellos y otros contribuyeron al debate político de la época sobre el acceso a la salud; hecho que se liga a las luchas por un cambio en América Latina, representadas en Ernesto Guevara, en la medicina, Paulo Freire en la Pedagogía o Camilo Torres Restrepo, Hélder Cámara, Pedro Casaldáliga, Leonardo Boff, Enrique Dussel en la Teología y filosofía de la Liberación, y otros que han influenciado la utilización las metodologías cualitativas en la investigación en salud. La investigación cualitativa en salud está fuertemente influenciada, como afirma Francisco Mercado (Mercado2002:18), por tres movimientos: la medicina social, los movimientos de base y los estudios socioculturales que construyen la investigación transdisciplinar en salud.

Visiones alternativas como el "Desarrollo a Escala Humana” (MaxNeef et alia, Chile), cuestionan los resultados del “desarrollo” en la mayoría de la población mundial; reclaman un desarrollo distinto donde primero estén las personas y se resten las crecientes y aterradoras diferencias e inequidades entre y dentro de los países, más evidentes si se comparan las condiciones de salud, como lo afirma Amartya Sen (Sen 2002:26), premio nobel de economía. De estas visiones se desarrollan propuestas como las de Elizalde (Elizalde 2006:10) y Escobar (Escobar 2005:13), que integran investigaciones en Física, Biología y ecología derivadas de Gregory Bateson, Berman, Capra, Spretnak y de los biólogos chilenos Maturana y Varela, ya que se trata de construir los conceptos de Salud y Desarrollo que reconocen a los servicios sanitarios son solo una parte de los elementos que determinan la vida de las personas (como afirma Vicente Navarro al definir "política nacional de salud" en www.vnavarro.org); lo que da sentido a campos transdisciplinares de la salud: Salud Pública, Salud Colectiva o Medicina Social. Transdisciplinariedad reconocida por Héctor Abad Gómez en 1987 antes de su asesinato (Abad 1987:1), y por Mario Chaves y Almeida en 1998 (Chaves 1998:8) ratificada luego de importantes avances en 2006 (Almeida-Filho 2006:3).

\section{Experiencias de APS en América Latina}

Las experiencias de APS en América Latina son numerosas y pese a su importancia son poco conocidas. Se reconocen especialmente, las que son fruto de cooperación internacional gubernamental y no gubernamental. Las experiencias van desde Centro América hasta la Patagonia, realizándose durante décadas antes de Alma Ata, como por ejemplo: en los 50’s, el “Hospital sin Paredes” (Hospital Carlos Luis Valverde de San Ramón) en Costa Rica, liderada por el doctor Juan Guillermo Ortiz; en México, las de Morones Prieto que inspiraron las promotoras rurales de salud en Colombia (como agentes comunitarios de salud), llevada a cabo por Abad Gómez (Cataño 1989:7) en la Universidad de Antioquia hasta ser un programa oficial a nivel nacional (donde se descubre la influencia sobre Abad de Milton Roemer, destacado salubrista discípulo de Sigerist y profesor de la Escuela 
de Salud Pública de la Universidad de California), las lideradas en la Universidad del Valle por Santiago Rengifo Salcedo (discípulo de Sigerist).

Cuba pese a sus limitaciones, es ampliamente reconocida tanto por sus logros sanitarios (Chelala1 1998:9) como por el desarrollo de la APS, sin embargo en la historia de ésta, el papel del médico argentino Ernesto Guevara es prácticamente excluido al igual que muchos cubanos; hecho explicable por el contexto de guerra fría que afectó la libertad académica en varios países Latinoamericanos ocultando gestores y experiencias valiosas como la cubana. La libertad universitaria se recuperó en Brasil después de la dictadura, pero en los otros países, con los años, la privatización de la educación superior la hizo presa de los mercados neoliberales, debilitando y acallando las voces criticas del pensamiento y la investigación. La Universidad Pública Brasilera es hoy líder en la región, se destaca en APS y por desarrollos en medicina social, trascendiendo con nombres como Sergio Arouca y logrando una campo de investigación reconocido como Salud Colectiva (Almeida-Filho 2006:3), con participación de académicos Argentinos y Chilenos con quienes se desarrollan postgrados de cooperación académica como por ejemplo la Escuela de Salud Publica de Chile o la Universidad de Lanus lugar de edición de la Colección de Libros Salud Colectiva y la Revista Salud Colectiva donde participan Mario Testa y Hugo Spinelli.

En el transcurso hasta aquí recorrido, Jaime Gofin (Gofin 2006:15), médico uruguayo, reconoce a tres pioneros de la Medicina Social y de la Atención Primaria en el mundo, a quienes conoció directamente y les asigna con claridad un papel fundamental en la APS y en la "Salud para Todos" a Guevara, Allende y al surafricano Sidney Kark. Gofin vive, lo que el siquiatra chileno Luis Weinstein, discípulo de Gustavo Molina, ha llamado en el paradigma transdisciplinar de la salud: la convergencia (Weinstein 2006:33).

En los 60 y 70 se destacan las experiencias de Carlos Alberto Alvarado y Elsa Moreno, en zonas rurales de Argentina derivadas del liderazgo de Ramón Carrillo, calificado por Mario Testa, como el sanitarista más importante de Argentina, Ministro de Salud, fallecido durante su exilio en Brasil en 1956. Las experiencias consistieron en organizar redes de atención entre Centros de Salud y Hospitales, articuladas con agentes comunitarios de salud para dar respuesta a la atención en grandes zonas geográficas, con población dispersa, con escasos profesionales y alejadas de las grandes ciudades que históricamente los concentran. La Dra. Moreno fue "heroína de América”, galardón de la Organización Panamericana de la Salud por su trabajo en programas de salud materno-infantiles; hoy trabaja en programas de formación en salud pública en este país. En Suramérica es destacada la experiencia Chilena con el movimiento pionero de medicina social latinoamericana, influenciado por Virchow y Sigerist, materializado en la generación de Allende (Waitzkin 2005:31) y Gustavo Molina Guzmán, influidos por su profesor de patología, alumno de Virchow, el doctor Max Westenhofer (Ibid 2005:32), prestigioso patólogo y científico de la evolu- 
ción. Allende Ministro de Salud en 1939 a los 29 años, escribió "La realidad medico social chilena” (Allende 1939:2). Molina, alumno de Sigerist en la Universidad de John Hopkins, es ejemplo de firmeza y compromiso, traductor y editor de textos que reúnen de manera selecta, trabajos muy representativos del pensamiento de Sigerist. Traducción realizada con sus compañeros de prisión en la "cárcel de los doctores" durante el golpe militar, antología que fue publicada durante su exilio en Colombia en 1974. Este texto es reeditado en Colombia en 2007, en conmemoración de los 50 años de la Muerte de Sigerist y de los 20 años de la muerte de Abad, la primera edición chilena es de 2008 como parte del Homenaje que realizó la que hoy es la Escuela de Salud Pública "Salvador Allende" al doctor Gustavo Molina, incluyendo la presentación de su hijo, también médico dedicado a la Salud Ocupacional, área que también promovió Molina padre.

Al tiempo que crecía la medicina integral cubana, derivada de la experiencia Chilena que además incluía la primera ley de salud ocupacional de América asociada a la lucha minera con Emilio Recabarren; la regionalización de servicios de salud para garantizar servicios completos en zonas geográficas determinadas, el desarrollo de centros o consultorios de atención primaria como el de Quinta Normal, con especialidades médicas y otros profesionales fuera del hospital, la escuela de Salud Pública de Chile, una las más importantes en América Latina, influenciando a toda la región, hasta el golpe militar que impidió que sus más brillantes representantes estuvieran en Alma Ata; sin duda, fueron de los principales ausentes de esta reunión. Los cambios sociales que se reclamaban en América Latina fueron acallados con terror y muerte bajo las dictaduras apoyadas por los gobiernos de EUA. La Facultad de Medicina y la Escuela de Salud Pública de Chile contaba con un número de destacados salubristas entre ellos Hugo Behn, director de la escuela en 1973 y exiliado en Costa Rica, Mariano Requena, los pediatras y salubristas Carlos Montoya Aguilar (jubilado de la OMS), Patricio Hevia actual director de la Unidad de Patrimonio Cultural de la Salud del Ministerio de Salud de Chile, Giorgio Solimano actual director de la Escuela de Salud Publica de Chile, y otros con su compromiso social alrededor de Allende, como Carlos Montoya subsecretario de Salud, ginecólogo y hoy historiador vinculado a la Unidad de Patrimonio, y el cirujano Arturo Jirón Vargas, Ministro de Salud en el Gobierno del presidente Allende, exiliado en Venezuela, que a su regreso continuó su trabajo universitario como cirujano en la Universidad de Chile, contribuyendo junto a otros colaboradores de Allende, para que su obra no sea olvidada, esfuerzos materializados en la Fundación Salvador Allende (www.fundacionsalvadoralllende.org).

\section{APS y las Experiencias Hermanas de Chile y Colombia}

Gustavo Molina, exiliado en Colombia fue docente de la Escuela Nacional de Salud Publica en la Universidad de Antioquia, desde donde promovió y desarrolló experiencias similares a las chilenas en la Medicina Integral y la integración de los servicios; se destaca la Integración Opera- 
cional de Abajo hacia Arriba (IOAPA), para el sector urbano (Medellín) y para el rural (Carmen de Viboral), que encontró ambiente propicio en el reciente Sistema Nacional de Salud de 1975. Desde esta escuela publicó artículos y un libro, documentos desconocidos y poco difundidos (Carvajal 2008:6), incluso en esta época de “renovación” de la APS. Gustavo Molina Guzmán pudo tener un papel protagónico en la historia de APS luego de Alma Ata, pero falleció en Medellín semanas antes de dicha reunión. Molina y Allende estuvieron vinculados a la larga lucha socialista democrática, cuyas conquistas fueron afectadas por el golpe y la dictadura militar que permaneció hasta los 90's razón por la cual, los logros alcanzados en Chile no se vieron en Alma Ata y hasta hoy, siguen bajo matices en el desarrollo de la reforma neoliberal que descentralizó y privatizó aunque no desmantelo totalmente el sistema público a diferencia de lo sucedido en Colombia. La reforma chilena se ha contenido débilmente con el Plan AUGE, los hospitales universitarios que siguen dependiendo del Ministerio de Educación y no proscribió la atención primaria; mientras en Colombia ni se la menciona hasta la ley 1122 de 2007 (donde apenas se nombra), que modifica la ley 100 de 1993 y que contrariamente, consolida el modelo del aseguramiento donde se "adquieren” paquetes según el nivel económico de las personas y con tal nivel de intermediación, que las personas, aunque tengan un carné, no pueden usarlo realmente.

\section{APS y Colombia Hoy y Ayer}

El caso colombiano tiene importancia internacional, hoy por ejemplo, se destaca la funesta aplicación de la formula neoliberal (Homedes, Ugalde 2005:16); antes, por las múltiples experiencias de APS incluso con concepciones anteriores a Alma Ata, especialmente en 1949 con el establecimiento del servicio Social obligatorio para profesionales de Salud promovido por Héctor Abad Gómez; pilares del Sistema Nacional de Salud especialmente en las zonas alejadas y rurales; con programas de varios centros académicos como la Facultad de Medicina de la Universidad del Valle, el Programa de Investigación en Modelos de Prestación de Servicios de Salud (PRIMOS), el Centro de Investigaciones Multidisciplinarias para el Desarrollo (CIMDER) y particularmente, el Programa de Investigación y Desarrollo de Sistemas de Salud (PRIDES) (Minsalud 1978:17); de la misma manera, con experiencias antioqueñas implementadas por Héctor Abad y otros, experiencias de integración rural y urbana con la participación de médicos colombianos como Alfredo Turizo Callejas, y extranjeros, como el destacado salubrista chileno Gustavo Molina Guzmán. Proyectos de cooperación internacional como el Colombo Holandés que aprovechó, entre otras, las experiencias en zonas rurales de egresados de la Universidad Nacional, como el doctor Ismael Lara, lo cual propició una experiencia valiosa en actividades de salud pública a los médicos holandeses cooperantes (Engelkes 1989:11), PEM Engelkes, (Idem 1990:12) o First Muller (Muller 1991:21), quienes registran varios documentos de su trabajo de APS en Colombia. Sin duda un papel fundamental en el desarrollo de la Escuela Nacional de Salud Pública y de la Escuela de Salud Publica la Universidad 
del Valle Santiago Rengifo Salcedo discípulo de Sigerist y de César Uribe Piedrahita principal precursor de programas atención integral en comunidades rurales y marginadas y por tanto de la APS en Colombia. Su inmensa obra está olvidada.

Otra experiencia colombiana destacable desde la Medicina Social, es el papel del médico-patólogo Guillermo Ferguson, al que niega y menosprecia la historia de la Atención Primaria de Salud. La ley 100 de 1993 y la inadecuada aplicación de la ley 10 de 1990 agudizan la crisis y llevan, por ejemplo, al cierre del principal hospital público "Hospital Universitario San Juan de Dios”, que fuera defendido en los años 70 por estudiantes y profesores de la Universidad Nacional de Colombia, costándole el arresto al decano de la Facultad de Medicina Guillermo Ferguson, quien es acompañado en su defensa por otro luchador olvidado y, entonces Decano de la Facultad de Derecho, Eduardo Umaña Luna.

Los académicos han sido victimas de interminables persecuciones que los han llevado al exilio y a otros a la muerte. El caso de Eduardo Umaña Mendoza (hijo) asesinado, y de Eduardo Umaña Luna (Padre), obligado al exilio dentro y fuera del país. Los más de 30 libros del profesor Umaña Luna son de escasa circulación pese a su importancia en la conceptualización y materialización de los derechos. Igual el caso de Salubristas e intelectuales objetivo de la violencia trágica del país y de Latinoamérica, aplazando los sueños por sociedades más justas, así como el movimiento medico-social chileno que fue perseguido y torturado por la dictadura de Pinochet.

En los años 90, previo al olvido de la APS, se la adoptó como política de salud en el Departamento del Valle, en esos años también se habló de APS en las grandes ciudades colombianas, con eventos que contaron con la participación del sector privado como la Fundación para la Educación Superior FES, por ejemplo, la olvidada Declaración de Bogotá, firmada por los Alcaldes de Bogotá, Cali, Bucaramanga y Medellín; se impulsaron enfoques unidos a la Promoción de Salud en el Seminario Nacional de “Atención Primaria y Promoción de la Salud”, realizado en Pereira en Septiembre de 1991, donde se propuso la conformación de la Red Nacional de Atención Primaria en Salud. Llama la atención el desconocimiento de la "Declaración de Bogotá” de 1990 por quienes recientemente han liderado políticas de salud para retomar la APS en las ciudades como en Bogotá (Vega, et al2008: 25) o Departamentos que participaron en el Encuentro Nacional de Modelos de Atención basados en Atención Primaria de Salud en el marco del Sistema de Seguridad Social en Salud, dado en Bogotá en mayo de 2005, reunión que hizo parte del proceso de renovación de la APS liderado por la OPS. Debe destacarse en 1992, la Conferencia de Promoción de Salud y Equidad de Bogotá, liderada por Helena Espinosa de Restrepo (alumna de Santiago Rengifo), apoyada por el Ministerio de Salud de Colombia, con la participación de países latinoamericanos. Eventos que fueron seguidos de un "olvido" oficial y académico con la progresiva implementación de la ley 100 de 1993, hasta los años 2000, cuando lenta- 
mente y gracias a movimientos ciudadanos como el Movimiento Mundial por la Salud de los Pueblos con las Asambleas en India (2000) y Ecuador (2005), vuelve a ser fundamental el tema de Sistemas de Salud y derecho a la salud, incluso impulsados por la OPS y la OMS, debido a los aniversarios 25 de Alma Ata en la Conferencia Internacional de Madrid y al aniversario 30, con los grupos regionales de trabajo en APS. Sus documentos han sido difundidos desde marzo de 2005 e incluyen otros de balance, particularmente el elaborado por la Escuela de Salud Pública de la Universidad de Chile (OPS-ESP UdeChile 2003:24), sobre la APS como política de salud. En este sentido también son importantes los aportes de la Comisión de Determinantes Sociales de la Salud cuyo informe final entregado en agosto de 2008, señala a la APS como núcleo de los sistemas de salud de los países y el Informe Mundial de OMS de 2008 que señala en su título "La APS Hoy más necesaria que nunca”. Es un claro resurgimiento de la APS y la Medicina Social en el mundo y en América Latina; el mundo debe cambiar y la Salud para Todos no puede ser más una realidad aplazada. 
Polis, Revista de la Universidad Bolivariana, Volumen 9, $N^{\circ}$ 27, 2010

\section{Nota}

* Apartes de la Tesis para optar al titulo de Doctor en Salud Pública en la Universidad Nacional de Colombia. 


\section{Bibliografía}

Abad Gómez, H. (1987), “Teoría y Práctica de Salud Pública”, Universidad de Antioquia, Medellín.

Allende, S. (1939), La Realidad Medico-Social Chilena, Ministerio de Salubridad. Santiago. [Soto, H., Requena, M. e Illanes, A. (1999), Reedición, Cuarto Propio, Santiago].

Almeida-Filho, N. (2006), "Complejidad y Transdisciplinariedad en el Campo de la Salud” en Salud Colectiva, vol.2, año 2, Buenos Aires

Basaglia, F. et al. (1978), Razón, Locura y Sociedad, Siglo XXI Editores, México.

Brown, T. y Fee E. (2002). "Sidney Kark and John Cassel Social Medicine Pioneers and South African Emigres” en American Journal of Public Health. Vol. 92 No.11.

Carvajal, Y. (2008), "Prólogo" en "Selecciones de Historia y Sociología de la Medicina” de Sigerist, H, Editorial Universidad Bolivariana, Santiago.

Cataño, L. O. (1989), “Héctor Abad Gómez, Promotor de la Salud” en Una vida por la vida, Juten Paul -Compilador, Ecoe, Bogotá.

Chaves M. (1998) “Complejidad y transdisciplinaridad un abordaje multidimensional” en Revista Brasilera de Educación Médica, Vol. 22 $\mathrm{N}^{\circ} 1$.

Chelala, C. (1998), “Cuba show health despite embargo” en BMJ, No. 3 London.

Elizalde, A. (2006), Desarrollo Humano y Ética para la Sustentabilidad, Universidad de Antioquia, Medellín.

Engelkes, E. (1990), “Process Evaluation In A Colombian Primary Health Care Programme” en Health Policy And Planning, Vol.5 año 4, London.

Ídem (1989), Health for All? Evaluation and Monitoring in a Comprehensive Primary Health Care project in Colombia, Royal Tropical Institute, Amsterdam.

Escobar, A. (2005), Más Allá del Tercer Mundo. Globalización y Diferencia, Instituto Colombiano de Antropología e Historia, Imprenta Nacional, Bogotá.

Frank, J. P. (1941), “The people’s misery: Mother of disease traslated of latin and introduction by Henry Sigerist” en Bulletin of the History of Medicine, No. 9, Baltimore. 
Gofin, J. (2006), “Acerca de 'Una Práctica de Medicina Social’ escrito por Emily y Sidney Kark” en Revista Medicina Social, No. 1:(2), New York.

Homedes, N. y Ugalde, A. (2005), "Why neoliberal health reforms have failed in Latin America” en Health Policy, Vol. 71 año 1, London.

Litsios, S. (2002), “The long and difficult road to Alma Ata: A personal reflection” en International Journal of Public Health, $\mathrm{N}^{\circ} 32$, Baltimore.

Mercado, F. (2002), “Qualitative research in Latin America: Critical perspectives on health” en International Journal of Cualitative Methods, supl.1, Edmonton.

Ministerio de Salud, Dirección General de Investigaciones (1978), Memorias Primera Reunión Nacional sobre Programas de Investigación en Planificación de Salud Cartagena 1977, Min. Salud, Bogotá.

Molina Bustos, C. (2006), “Antecedentes del Servicio Nacional de Salud: Historia de Debates y Contradicciones Chile 1932-1952” en "Cuad. Med. Soc., Vol. 46 año 4, Santiago.

Muller, F. (1991), Pobreza, Participación y salud: casos latinoamericanos, Universidad de Antioquia, Medellín.

Navarro, V. (1984), Lucha de Clases, Estado y Medicina, Nueva Imagen, México.

OPS - Escuela de Salud Pública Universidad de Chile (2003), Revisión de las Políticas de Atención Primaria de Salud en América Latina y el Caribe. Informe final, Santiago.

Paul B. H. (1955), Culture and Community: Case Studies of reactions to health Programs, Russell Sage Foundation, New York.

Rodríguez, L. (19979, “Cesar Uribe Piedrahita, un científico fuera de serie” en Rev. Acad. Colom. Cienc. Vol. 21, N 80.

Sen, A. (2002), “¿Por qué la equidad en Salud?” en Rev. Panam. Salud Pública, $\mathrm{N}^{\circ}$. 11, Washington.

Sigerist, H. (2006), “Johann Peter Frank: Un pionero de la Medicina Social” en Salud Colectiva, Vol. 2, año 3, Buenos Aires.

Ídem (2003), “Medical Care for All. Voices from the Past” en American Journal of Public Health, Vol. $93 \mathrm{~N}^{\circ}$ 1, Stanford.

Ídem (1943), "From Bismarck to Beveridge. Developments and tends in Social Segurity legislation” en Bulletin of History of Medicine, Vol. $13 \mathrm{~N}^{\circ}$ 4, Baltimore. 
Vega R., Acosta N., Mosquera P. y Restrepo M. (2008), “La Política de Salud en Bogotá 2004-2008. Análisis de la Experiencia de Atención Primaria Integral de Salud” en Revista Medicina Social, Vol. 3 año 2, New York.

Waitzkin, H. (2006), "Un Siglo y medio de olvidos y redescubrimientos: las perdurables contribuciones de Virchow a la Medicina Social” en Medicina Social, Vol. 1, año 1, New York.

Ídem (2005), “Commentary: Salvador Allende and the bird of Latin American social medicine” en International Journal of Epidemiology, Vol. $34 \mathrm{~N}^{\circ} 4$ Oxford.

Weinstein, L. (2006), Hacia el Homo Sapiens. El encuentro del asombro, la poesía y el paradigma de la integración, Editorial Universidad Bolivariana, Santiago.

Recibido: 09.06.2009

Aceptado: 03.02.2010 